\title{
Heat transfer enhancement in micro-channels caused by vortex promoters
}

\author{
M. Meis , F. Varas ，A. Velázquez , J.M. Vega \\ Applied Mathematics Department, School of Telecommunication Engineering, Universidad de Vigo, Campus Marcosende, 36310 Vigo, Spain \\ Aerospace Propulsion and Fluid Mechanics Department, School of Aeronautics, Universidad Politécnica de Madrid, Plaza del Cardenal Cisneros 3, 28040 Madrid, Spain \\ Applied Mathematics Department, School of Aeronautics, Universidad Politécnica de Madrid, Plaza del Cardenal Cisneros 3, 28040 Madrid, Spain
}

\section{A R T I C L E I N F O}

Keywords:

Micro-cooling systems

Vortex promoter

Micro-channel flow

Heat transfer

\begin{abstract}
A B S T R A C T
This paper presents a systematic numerical study of the effects of heat transfer and pressure drop produced by vortex promoters of various shapes in a 2D, laminar flow in a micro-channel. The liquid is assumed to be water, with temperature dependent viscosity and thermal conductivity. It is intended to obtain useful design criteria of micro-cooling systems, taking into account that practical solutions should be both thermally efficient and not expensive in terms of the pumping power. Three reference cross sections, namely circular/elliptical, rectangular, and triangular, at various aspect ratios are considered. The effect of the blockage ratio, the Reynolds number, and the relative position and orientation of the obstacle are also studied. Some design guidelines based on two figures of merit (related to thermal efficiency and pressure drop, respectively), which could be used in an engineering environment are provided.
\end{abstract}

\section{Introduction}

Micro-channel flow has been extensively studied during the last decade because of its interest from both the scientific and the technological point of view. On the scientific side, laminar flow in ducts with hydraulic diameters ranging from tens to hundredths of microns presents some distinctive features that are still being discussed and remain somewhat controversial. One of the main open issues is whether the observed high heat transfer rates are of a fluid dynamics origin or are due to other reasons, such as rarefaction and electro-kinetic effects, which in turn have been extensively studied in this context in the specialized literature, as the interested reader can attest.

On the technological side, micro-channel flow exhibits practical applications with a strong impact in the conceptual design of many systems/subsystems that are relevant in various industrial sectors. For example, making more compact and lighter designs is very attractive in aerospace engineering because, in the end, it translates into economic gains. Weight and space are at a premium in modern aircraft design, and micro-heat exchangers are the ideal candidates to perform the thermal control of a new generation of high capability avionics characterized by the large heat dissipation.

In connection with specific aerospace engineering applications, both passive and active micro-cooling systems are currently being taken into consideration. Broadly speaking, passive systems (based on phase change) do not require an external pump and thus tend to exhibit both a low maintainability cost and a long working life, which make them well suited for civil applications. If thermal efficiency is the main priority, as it happens in defence applications, active systems might be preferred, even though in this case the designer should not forget pressure drop, to avoid increasing too much the power of the pump. Nevertheless, the distinction between civil and defence applications is being blurred as industrial contractors struggle to cope with budgets that (if not actually decreasing) are becoming more and more demanding. In this context, optimization of micro-cooling systems behavior is an issue that may have a non-negligible economic impact.

Concerning heat transfer in micro-channel cooling systems, it must be kept in mind that steady, laminar flow in a standard channel is almost parallel (non-parallelism being only due to dependence of viscosity on temperature) which means that thermal conductivity (which is quite low in ordinary liquids) is the only mechanism for heat transport from the hot wall to the bulk. Nevertheless, heat transfer in these systems can be enhanced by vortex promoters (built-in obstacles in the micro-channels), since they produce transversal convection, which is a quite effective heat transport mechanism if the produced vortices exhibit appropriate size and intensity.

Channel flow heat transfer (both from the channel walls and from the vortex promoter itself) has been addressed in a number of papers. The case of a square vortex promoter has been considered by Rahnama and Moghaddam [1], who obtained a correlation between Nusselt and Reynolds numbers, and by Turki et al. [2] who addressed mixed convection and generated engineering correla- 
tions between the relevant parameters of the problem. Icoz and Jaluria [3] considered a rectangular channel with a transversal aspect ratio of 6 to constrain 3D effects to the wall regions. The vortex promoters had circular, square, and hexagonal shapes, and were located, with several blockage ratios, ahead of two tandem heating sources. The authors reported that the hexagonal shape is best to remove heat from the first heat source and the circular one is optimal when heat removal from the second source is sought; the square vortex promoter instead is the best candidate when looking for a reasonable combination of heat transfer and pressure drop. The case of a triangular vortex promoter has been studied in detail by Abbasi and co-workers [4,5], who reported that the vortex promoter produced an $85 \%$ increase in the time averaged Nusselt number at Reynolds 250. Nitin and Chhabra [6] considered a rectangular vortex promoter immersed in a non-Newtonian fluid, and reported heat transfer variations of the order of $10 \%$ and a strong sensitivity on the power law used to describe non-Newtonian behaviour.

The turbulent regime (Reynolds number larger than, say, 2000) has been analysed by Valencia [7], who reported a heat transfer enhancement of the order of $30 \%$, with a fivefold increase in the friction factor. Note that turbulence promotes mixing in all scales ranging from the Kolmogorov scale to the diameter of the channel, but only the larger scales are really effective to enhance cooling; small scales have almost no effect on cooling and produce a large viscous dissipation that highly increases the pressure drop.

The use of tandem cylinders as mixing promoters is also the current subject of much research interest. Fluid dynamic issues have been studied by Papaioannou et al. $[8,9]$ and Tasaka et al. [10], while heat transfer effects have been considered by Niu et al. [11] and by Valencia and co-workers [12-14].

In spite of this background on heat transfer enhancement by vortex promoters in channel flow, elaboration of specific design guidelines in cooling micro-systems has received little attention. In this sense, it must be kept in mind that micro-manufacturing costs must be considered as a key factor (for instance, complex vortex promoter geometries as proposed in [3] might be unaffordable) and liquids are used as cooling fluids, whose viscosity and thermal conductivity are both temperature-dependent. However, all the references mentioned above consider air as the cooling fluid, and existing literature using water (e.g., [15]) concentrates on heat transfer from/to the obstacle.

In this paper, we consider the case of a single obstacle with various cross section shapes, namely triangular, rectangular, and elliptical, and aspect ratios ranging from $1 / 1$ to $1 / 16$. In addition, we vary the blockage ratio, the position and orientation of the obstacle, and the Reynolds number. With all these, we intend to provide some engineering guidelines to select mixing promoters for microcooling systems. For reasons explained above, we consider both the heat transfer rate enhancement and the pressure drop increase. To ensure that our predictions are as close as possible to practical applications, we account for temperature dependent fluid viscosity and thermal conductivity.

The remaining of the paper is organized as follows. The problem is formulated and the numerical method is described in Section 2, with some details relegated to Appendix A, at the end of the paper. Dependence of the thermal efficiency and pressure drop on shape, aspect and blockage ratios, and position and orientation is analyzed in Section 3 at the fixed Reynolds number $R e=600$, based on the hydraulic diameter; larger Re are briefly considered in Section 4. And some concluding remarks are given in Section 5.

\section{Problem statement and solution method}

We consider the 2D, unsteady, laminar flow of water in a nonisothermal micro-channel. A cylindrical obstacle is placed crossflow wise inside the channel (Fig. 1). Walls are adiabatic except in a portion of the bottom wall located downstream of the obstacle where the temperature is maintained at $\widetilde{T}^{\text {max }}=353 \mathrm{~K}$ (with tildes denoting hereafter dimensional quantities), while the temperature of the incoming flow is $\widetilde{T}^{\text {inlet }}=293 \mathrm{~K}$. Such temperature variation implies that the viscosity varies by a factor of 3 [16] and leads us to take into account dependence of viscosity (and of thermal conductivity, which also varies significantly) on temperature. The spatial coordinates, $x$ and $y$, the velocity components, $u$ and $v$, and the pressure $p$ are made dimensionless using the hydraulic diameter of the channel $D_{h}$, (namely, twice the height of the channel, $2 \tilde{h}$ ) the velocity at the inlet section, $\tilde{u}^{\text {inlet }}$, and $\tilde{\rho}\left(\tilde{u}^{\text {inlet }}\right)^{2}$, respectively, where $\tilde{\rho}$ is the density, assumed constant; time is made dimensionless using the characteristic time $t_{c}=D_{h} / \tilde{u}^{\text {inlet }}$; nondimensional temperature is defined as $T=\left(\widetilde{T}-\widetilde{T}^{\text {inlet }}\right) /\left(\widetilde{T}^{\text {max }}-\widetilde{T}^{\text {inlet }}\right)$. The governing equations (continuity, momentum, and energy conservation) are

$$
\begin{aligned}
\partial_{x} u+\partial_{y} v=0, & \\
\partial_{t} u+u \partial_{x} u+v \partial_{y} u= & -\partial_{x} p+\frac{1}{\operatorname{Re}}\left[\partial_{x}\left(\mu \partial_{x} u\right)+\partial_{y}\left(\mu \partial_{y} u\right)\right. \\
& \left.+\partial_{x} \mu \partial_{x} u+\partial_{y} \mu \partial_{x} v\right], \\
\partial_{t} v+u \partial_{x} v+v \partial_{y} v= & -\partial_{y} p+\frac{1}{\operatorname{Re}}\left[\partial_{x}\left(\mu \partial_{x} v\right)+\partial_{y}\left(\mu \partial_{y} v\right)\right. \\
& \left.+\partial_{y} \mu \partial_{y} v+\partial_{x} \mu \partial_{y} u\right], \\
\partial_{t} T+u \partial_{x} T+v \partial_{y} T= & -\frac{1}{\operatorname{Re} \operatorname{Pr}}\left[\partial_{x}\left(\kappa \partial_{x} T\right)+\partial_{y}\left(\kappa \partial_{y} T\right)\right],
\end{aligned}
$$

where $\partial_{x}, \partial_{y}$, and $\partial_{t}$ denote the partial derivatives with respect to $x$, $y$, and $t$, respectively. The (inlet) Reynolds and Prandtl numbers are defined as

$\operatorname{Re}=D_{h} \tilde{\rho}^{\text {inlet }} \tilde{u}^{\text {inlet }} / \tilde{\mu}\left(\widetilde{T}^{\text {inlet }}\right), \quad \operatorname{Pr}=\tilde{c}_{p} \tilde{\mu}\left(\widetilde{T}^{\text {inlet }}\right) / \tilde{\kappa}\left(\widetilde{T}^{\text {inlet }}\right)$,

where $\tilde{\mu}, \tilde{\kappa}$, and $\tilde{c}_{p}$ are the viscosity, thermal conductivity, and the specific heat, respectively. Dependence of dimensionless viscosity $\mu$ and thermal conductivity $\kappa$ on temperature is assumed quadratic [16], namely

$\mu=\frac{\tilde{\mu}(\widetilde{T})}{\tilde{\mu}\left(\widetilde{T}^{\text {inlet }}\right)}=1-\mu_{1} T+\mu_{2} T^{2}, \quad \kappa=\frac{\tilde{\kappa}(\widetilde{T})}{\tilde{\kappa}\left(\widetilde{T}^{\text {inlet }}\right)}=1+\kappa_{1} T-\kappa_{2} T^{2}$,

where (in the temperature range $293 \mathrm{~K}<\widetilde{T}<353 \mathrm{~K}$ ) the various coefficients appearing in these expressions are

$\mu_{1}=1.1292, \quad \mu_{2}=0.4904, \quad \kappa_{1}=0.1572, \quad \kappa_{2}=0.0470$.

The boundary conditions (see Fig. 1) result from imposing a flat velocity profile at the inlet section (to somehow simulate the inlet

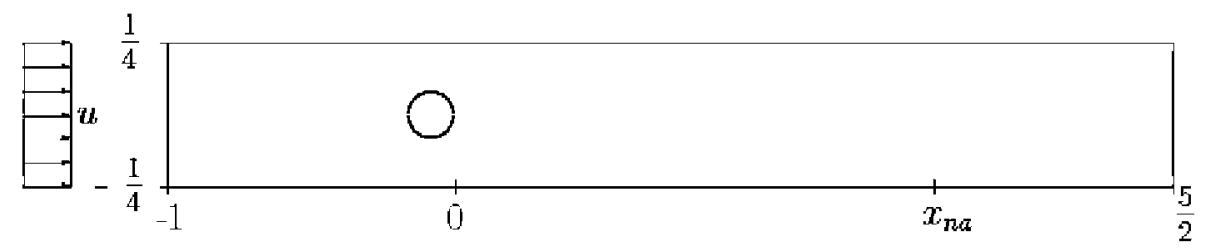

Fig. 1. Sketch of the computational domain. 
flow in the micro-channel coming from an stagnation chamber), namely

$u=1, \quad v=0, \quad T=0$

at $x=-1$, while at the outlet section, we require the absence of (total) stress and diffusive heat flux, namely

$2 \mu \partial_{x} u-p=\mu\left(\partial_{x} \nu+\partial_{y} u\right)=\partial_{x} T=0$

at $x=5 / 2$. In order to facilitate future experimental validation, heat transfer from the wall is assumed to occur only in a part of the wall, to be referred to the nonadiabatic part in the sequel, corresponding to $0<x<x_{\text {na }}, y=-1 / 4$ with $x_{\text {na }}=5 / 3$ (see Fig. 1 ). There, we impose no slip and a fixed, constant temperature, namely

$u=v=0, \quad T=1$,

while at the remaining part of the lower wall, at the upper wall, and at the obstacle (whose center coordinates will be denoted $x_{c}$ and $y_{c}$ ), we impose no slip and no heat flux, namely

$u=v=0, \quad \partial_{n} T=0$,

where $n$ is the unit normal to the surface.

Eqs. (1) - (4) with boundary conditions (8)-(11) and appropriate initial conditions are solved using the finite element solver COMSOL Multiphysics [17]. In particular, $P_{2}-P_{1}$ Taylor-Hood finite elements are used to discretize velocity components and pressure, and $P_{2}$ finite elements are chosen for temperature. Meshes are fine enough (some details are given in Appendix A, at the end of the paper) to avoid the need for stabilizing convective terms in momentum equations. Streamline-Upwind/PetrovGalerkin (SUPG) stabilization is used in the energy equation outside the thermal boundary layers to stabilize the thermal field in the wake.

In order to get an accurate computation of heat transfer from the wall (and avoid post-processing of the temperature field), heat flux in the nonadiabatic part of the lower wall is introduced as a Lagrange multiplier of the (thermal) boundary condition (by imposing this condition in weak form) and then discretized with $P_{1}$ finite elements defined on the boundary (higher order elements cannot be used, to avoid numerical instability).

Time integration is performed in COMSOL Multiphysics with a variable-step, variable-order implementation of backward differentiation formulae. Strict tolerances on (local) truncation errors in time integration are used (typical relative tolerances are always taken well below $10^{-3}$ ) so that the final error of the numerical solution is dominated by the spatial discretization error. A mesh dependency analysis is given in Appendix A.

In order to analyze thermal efficiency, we shall consider the local, time averaged heat transfer in the nonadiabatic portion of the lower wall, $\widetilde{Q}^{\prime}$, which can be measured by the local ( $x$-dependent) and global, time averaged Nusselt numbers,

$$
\begin{aligned}
& \langle N u\rangle=\frac{\widetilde{Q}^{\prime}}{2\left(\widetilde{T}^{\text {max }}-\widetilde{T}^{\text {inlet }}\right) \tilde{\kappa}\left(\widetilde{T}^{\text {max }}\right) \tilde{h}}=\frac{1}{t_{1}-t_{0}} \int_{t_{0}}^{t_{1}} \partial_{y} T(x,-1 / 4, t) d t, \\
& \langle N u\rangle^{\text {global }}=\int_{0}^{x_{n a}}\langle N u\rangle d x,
\end{aligned}
$$

where $t_{0}$ and $t_{1}$ are chosen large enough to avoid both transients and dependence of the time-average on the integration interval. Thermal efficiency $\Phi_{T}$ and mechanical penalty $\Phi_{M}$ are defined as

$\Phi_{T}=\frac{\langle N u\rangle^{\text {global }}}{\langle N u\rangle_{\text {ref }}^{\text {global }}}, \quad \Phi_{M}=\frac{\langle\Delta p\rangle^{\text {global }}}{\langle\Delta p\rangle_{\text {ref }}^{\text {global }}}$,

where the subscript ref stands for the reference configuration when no obstacle is present and $\langle\Delta p\rangle^{\text {global }}$ stands for the time averaged, total pressure drop, from entrance to exit. Because of the way we model the problem (the computational domain height is fixed while the obstacle size is varied), dimensionless pump power (volume flow times pressure drop) depends only on $\langle\Delta p\rangle^{\text {global }}$. This means that $\Phi_{M}$ is a direct measure of the power needed to propel the fluid along the channel, across the vortex promoter. In general, we shall intend to maximize $\Phi_{T}$, but mechanical penalty will also be taken into account.

\section{Results in the low Reynolds number regime}

We first select a Reynolds number of 600 based on the hydraulic diameter (twice the channel height, see Eq. (5)) and study the effect of the following properties of the configuration:

- Obstacle shape (circle/ellipse, rectangle, and triangle) and aspect ratio $\alpha=\tilde{d}_{h} / \tilde{d}_{v}$, where $\tilde{d}_{h}$ and $\tilde{d}_{v}$ are the horizontal and vertical sizes, when the obstacle is placed in symmetric position.

- Channel blockage ratio $\beta=\tilde{d}_{v} / \tilde{h}$, where $\tilde{h}$ is the channel height. - Obstacle orientation and position.

In order to analyze dependence on the blockage ratio, we consider a centered (at the origin) circular obstacle with the blockage ratio $\beta$ ranging from $1 / 10$ to $1 / 2$, which corresponds to Reynolds numbers based on the obstacle diameter varying from 30 to 150 , well inside the assumed 2D laminar regime. The flow is steady for $\beta<\beta_{c} \simeq 0.154$ and oscillatory for larger blockage ratio (except for values of the blockage ratio close to 1 , when the flow becomes steady again, see [18]). Such value of $\beta_{c}$ yields a value of the Reynolds number based on the obstacle diameter of 46.2 , which is roughly the same that the critical value for the appearance of the von Karman street in isothermal open flow around a circular obstacle (see $[18,19]$ ), as expected since the blockage ratio is quite small and nonisothermal effects are very weak on the obstacle. Nevertheless, it must be observed that in practice oscillatory solutions can appear at a lower Reynolds number due to obstacle vibration [20].

The vorticity snapshots in Fig. 2 show that the strength of the von Karman vortices increase as the blockage ratio increases, producing some transversal plumes at the interface between adjacent vortices, which are stronger near the (hotter) lower wall. In fact, the whole flow field is non-symmetric around the horizontal centerline, which is due to temperature dependence of viscosity and thermal conductivity and is more clearly appreciated in the whole series of vorticity snapshots, which are omitted here. When these plumes are strong enough, they are quite effective in enhancing heat transport from the (thermal boundary layer attached to the) hot wall to the bulk, as appreciated in the temperature contours plotted in Fig. 3. This thermal efficiency increase is further appreciated in Fig. 4, where both the time averaged, local Nusselt number and the thermal efficiency are plotted. Left plot shows that local Nusselt number diverges at $x=0$, which is due to the weak singularity that appears at the wall as the boundary condition changes from zero heat flux to constant temperature. As seen in left plot, the singularity plays a role in cooling enhancement at low blockage ratio, when vertical convection enhancement is localized near the obstacle itself. At larger $\beta$ instead the above mentioned transversal plumes come into play and heat transfer enhancement remains downstream. As a consequence, thermal efficiency increases as the vortices become stronger, which occurs as the blockage ratio increases. This can be seen in the right plot in Fig. 4, which shows that a circular obstacle produces only a moderate increase of thermal efficiency except for large blockage ratios, which in turn generate a large pressure drop.

Now, in order to analyze the effect of the obstacle shape, we consider two fixed blockage ratios, $\beta=1 / 3$ and $\beta=1 / 2$, and vary 


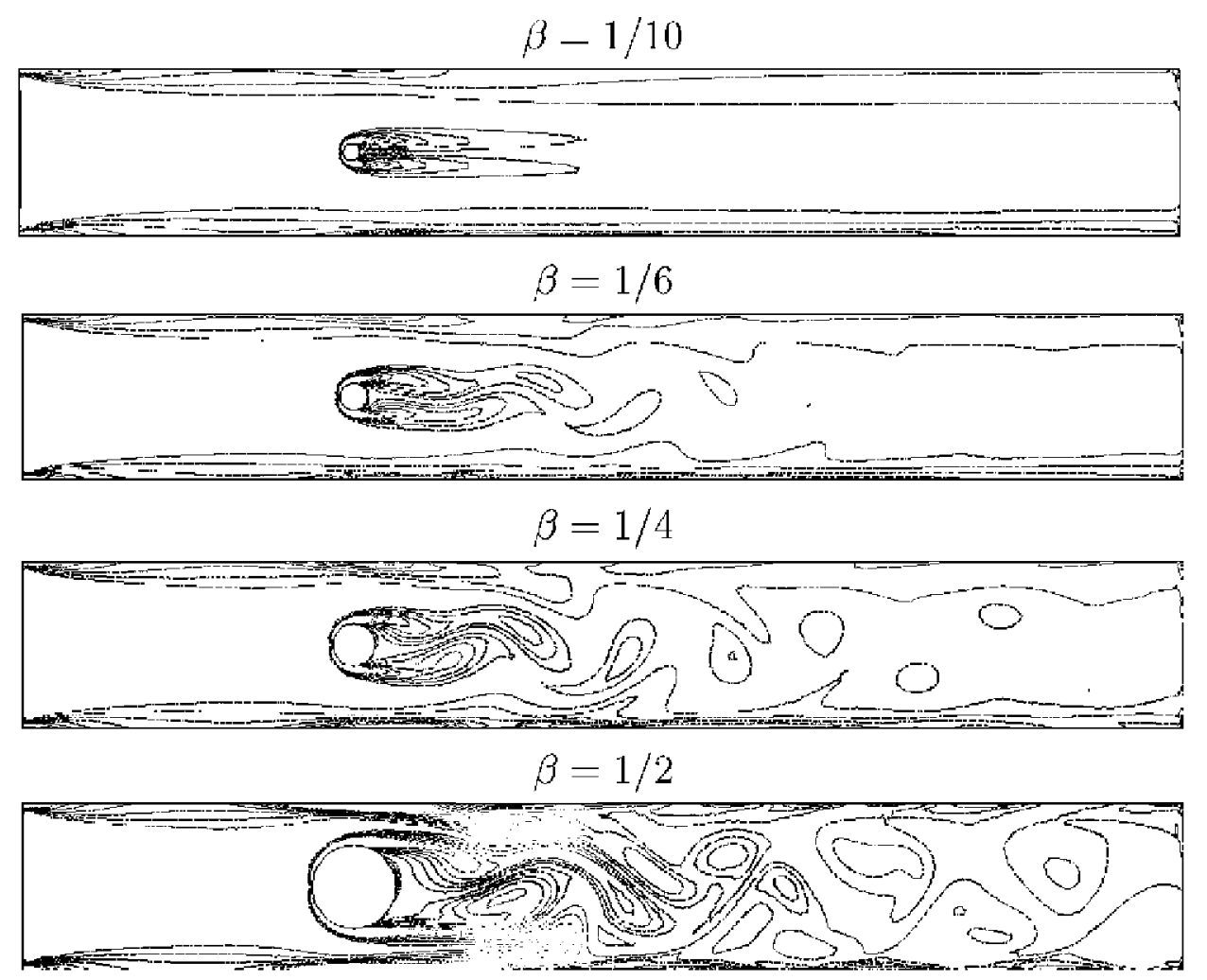

Fig. 2. Instantaneous (absolute value of) vorticity contours for a circular obstacle at the indicated blockage ratios and $\operatorname{Re}=600$.

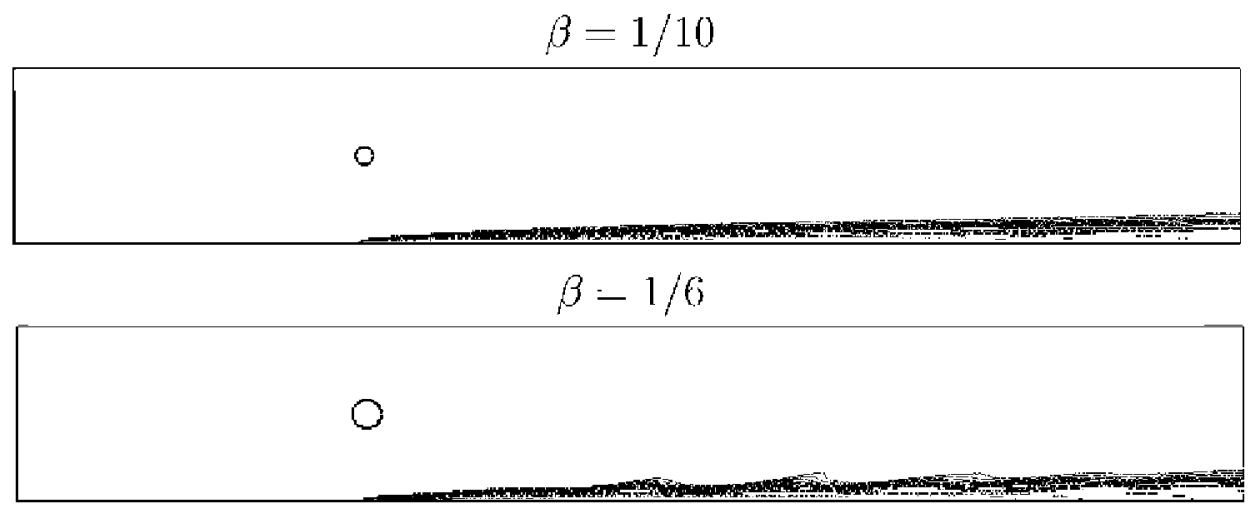

$$
\beta-1 / 4
$$

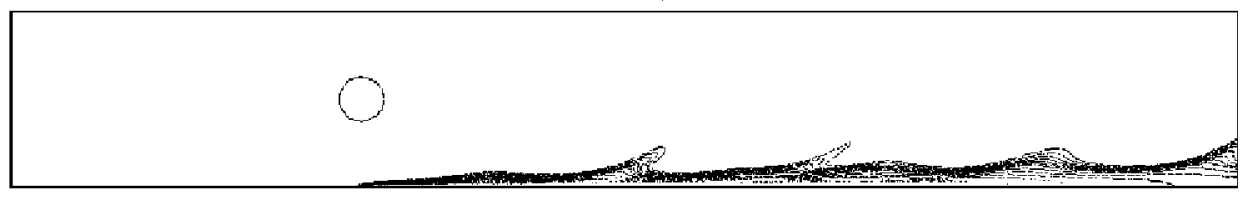

$$
\beta=1 / 2
$$

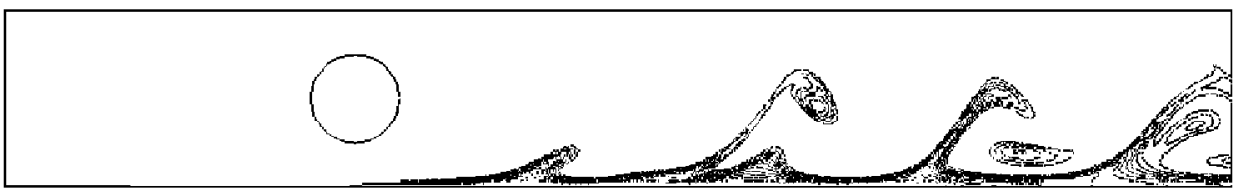

Fig. 3. Instantaneous temperature contours for a circular obstacle at the indicated blockage ratios and $\mathrm{Re}=600$.

both the aspect ratio and the obstacle shape; the obstacle is located at the center of the channel. Vorticity and temperature contours are qualitatively similar to those in Figs. 2 and 3, and are omitted; the counterpart of Fig. 4 instead is given in Figs. 5 and 6.
The main conclusions drawn from these results are:

- Increasing the blockage ratio and reducing the aspect ratio increases the global Nusselt number for all configurations. Incre- 

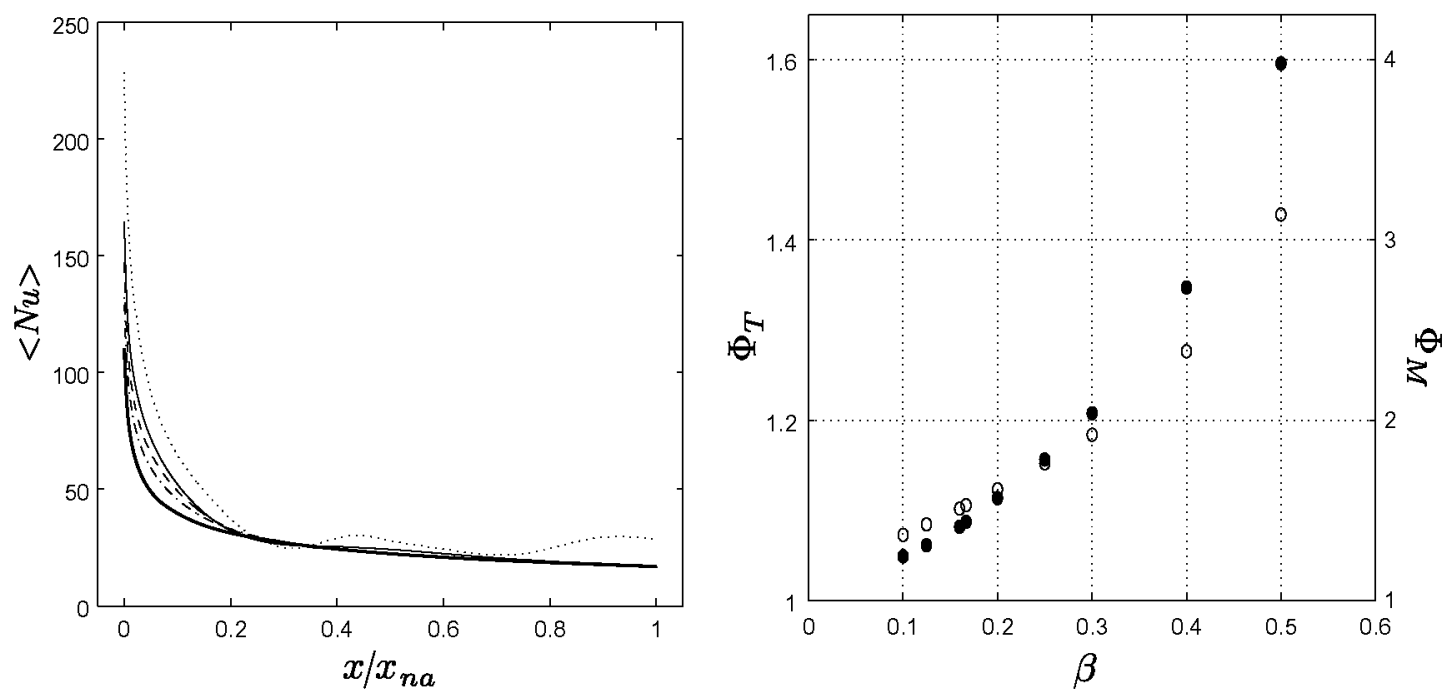

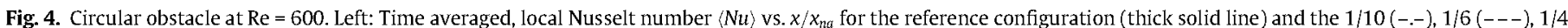
$(-)$, and $1 / 2(\cdots)$ blockage ratio cases. Right: Thermal efficiency $\Phi_{T}$ (plain circles) and mechanical penalty $\Phi_{M}$ (shaded circles) in terms of the blockage ratio.

ments up to $140 \%$ can be obtained for the smallest obstacle aspect ratio at $1 / 2$ blockage ratio.

- When looking at heat transfer effects, the triangle, no matter what its aspect ratio or its blockage ratio might be, is consistently better than the other configurations. Next comes the circle and, then, the rectangle. Additionally, heat transfer gain in the case of triangle vortex promoters is less sensitive to the aspect ratio.

- For all cases, any given increment on the Nusselt number comes at the expense of a larger pressure drop. This effect is stronger for obstacles having the smallest aspect ratios. In particular, the mechanical penalty $\Phi_{M}$ could be as high as 3.8 and 7.5 for the (1/16)-aspect ratio triangular obstacle at $\beta=1 / 3$ and $1 / 2$, respectively.

At the same time, when looking at guidelines for the design of practical cooling systems, the manufacturing cost is also a factor to be considered. For this reason, the circle and the rectangle appear to be the most promising selection because the micro-man- ufacturing of ellipses and triangles is trickier than that of circular (simple off-the-shelf micro-cables could do it) and/or rectangular obstacles. In the last case, aspect ratio should also be kept within some bounds (standard micro-machining techniques penalize sizes less than $50 \mu \mathrm{m}$ ). Also, when heat transfer and pressure drop are combined, the rectangle shows a peak performance at an aspect ratio of about $1 / 2$, which corresponds to a $100 \times 50 \mu \mathrm{m}^{2}$ obstacle in a typical micro-channel with a height of $300 \mu \mathrm{m}$, if $\beta=1 / 3$ (and a larger size if $\beta=1 / 2$ ). To study the effect of position and orientation of the vortex promoter, we shall concentrate on the rectangles with $\alpha=1 / 2$. These can somehow mimic a triangle shape when rotated. In addition, rectangles with $\alpha=1 / 10$ will be considered to illustrate the trends in heat transfer enhancement obtained by reducing the aspect ratio. As for the blockage ratio, the values $\beta=1 / 3$ and $\beta=1 / 2$ will be used. Thermal efficiency and mechanical penalty for these cases are plotted in Figs. 7-10 vs. the obstacle position (measured by the vertical position of the center) and orientation (measured by counterclockwise rotation). These results show that:
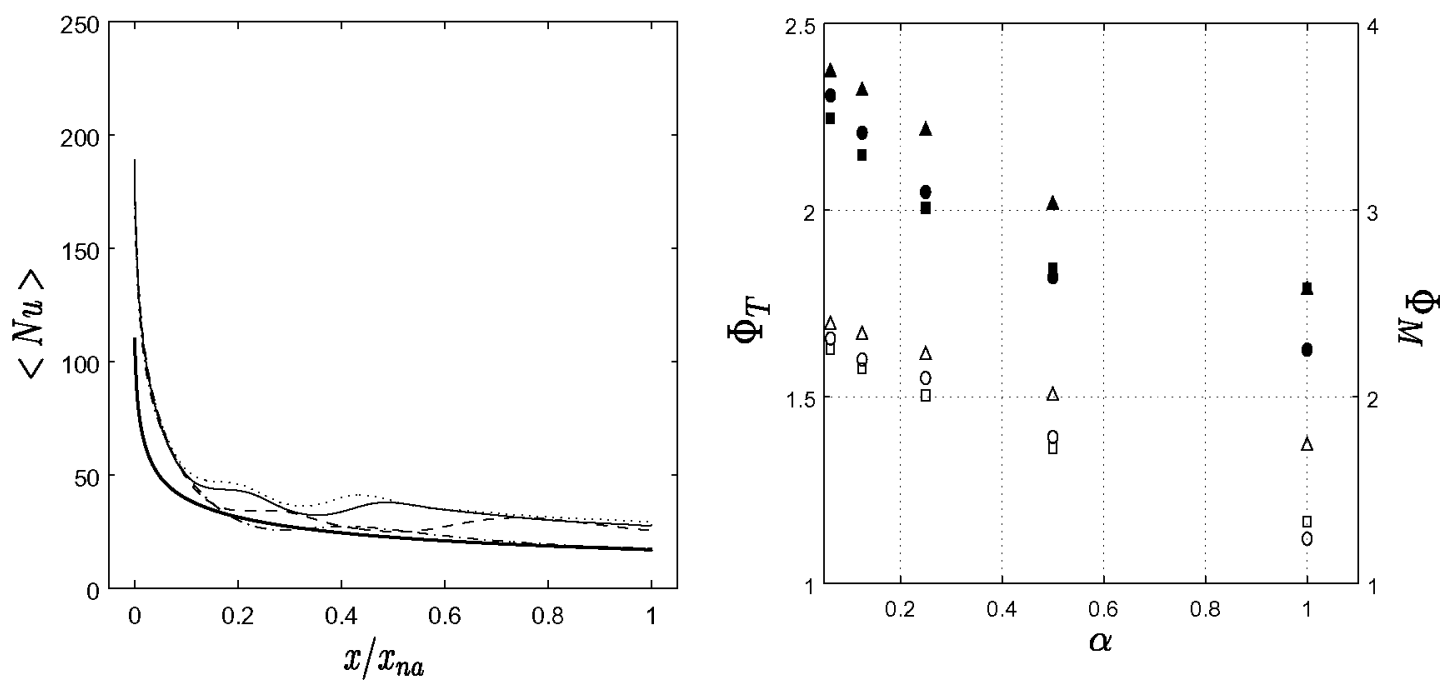

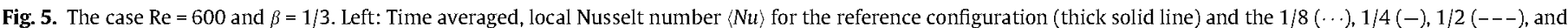

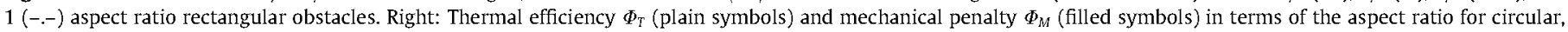
triangular, and rectangular obstacles. 

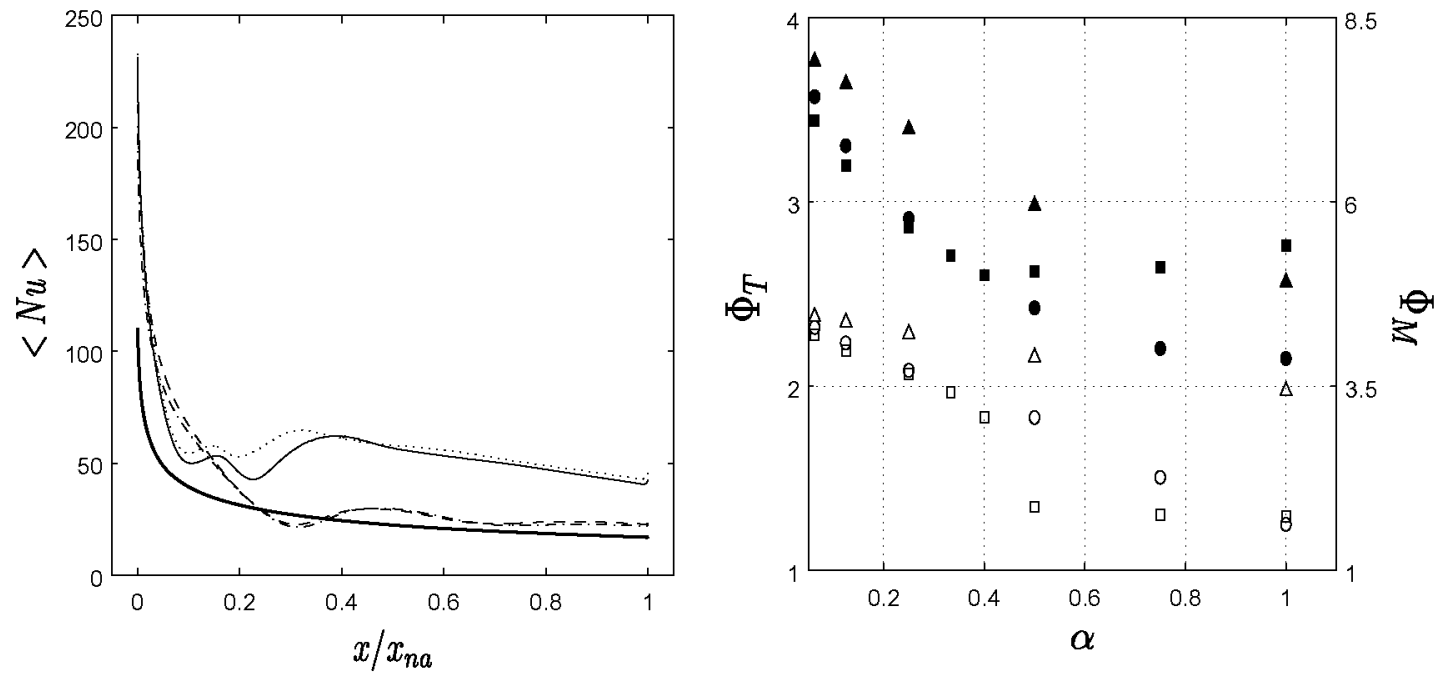

Fig. 6. As in Fig. 5 but for $\beta=1 / 2$.
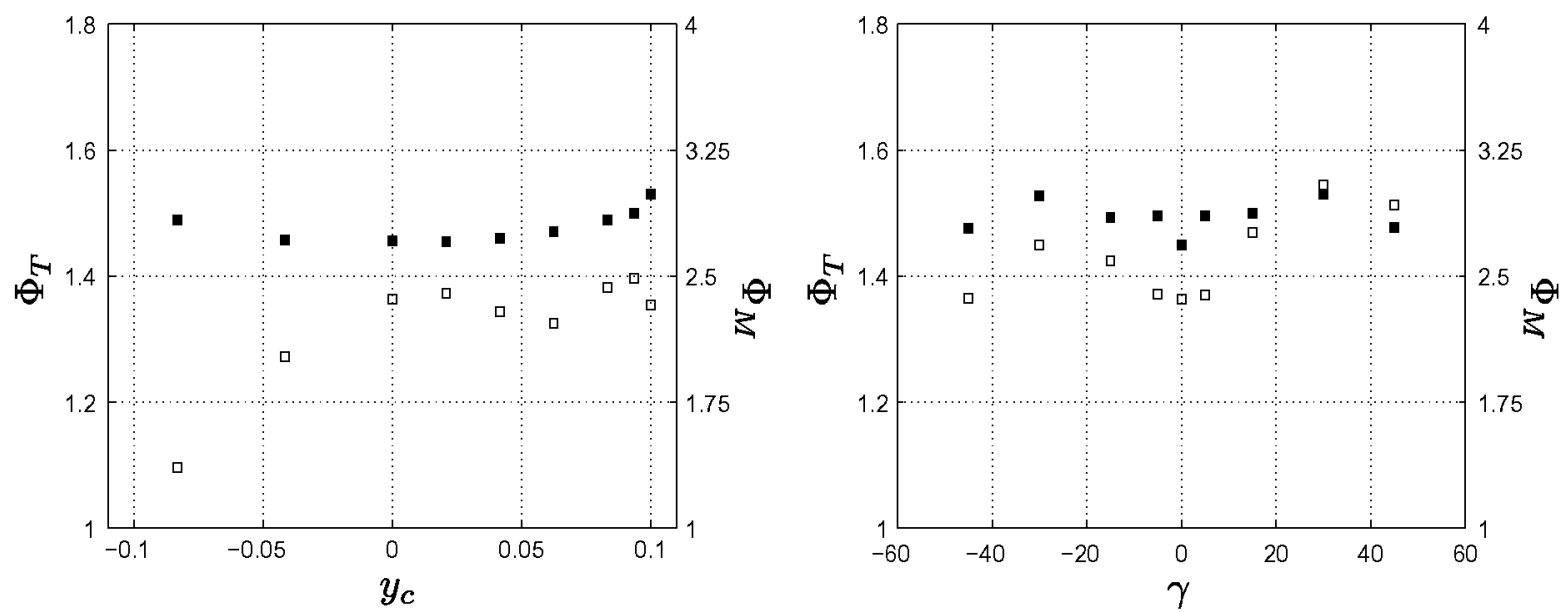

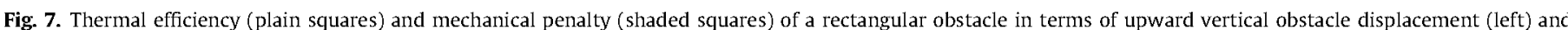
counter-clockwise obstacle rotation around its center (right) for $\alpha=1 / 2, \beta=1 / 3$, and $\operatorname{Re}=600$.
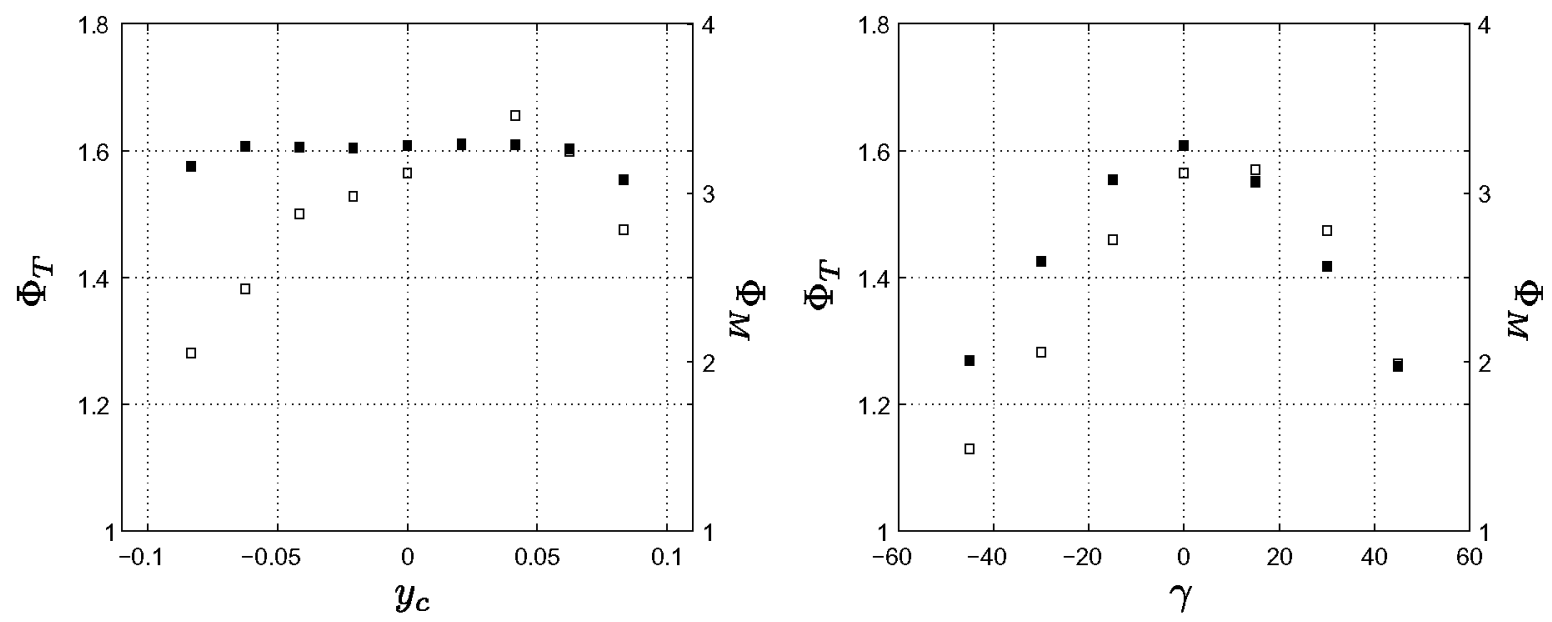

Fig. 8. As in Fig. 7, for $\alpha=1 / 10$ and $\beta=1 / 3$.

- Upward displacement at $\beta=1 / 3$ (Figs. 7 and 8, left) produce either no improvement (if $\alpha=1 / 2$ ) or a slight benefit (if $\alpha=1 / 10$ and the displacement is small), whereas thermal effi- ciency decreases significantly as the obstacle is moved downwards. The latter is due to the fact that the thermal efficiency enhancement that could be expected from approaching the vor- 

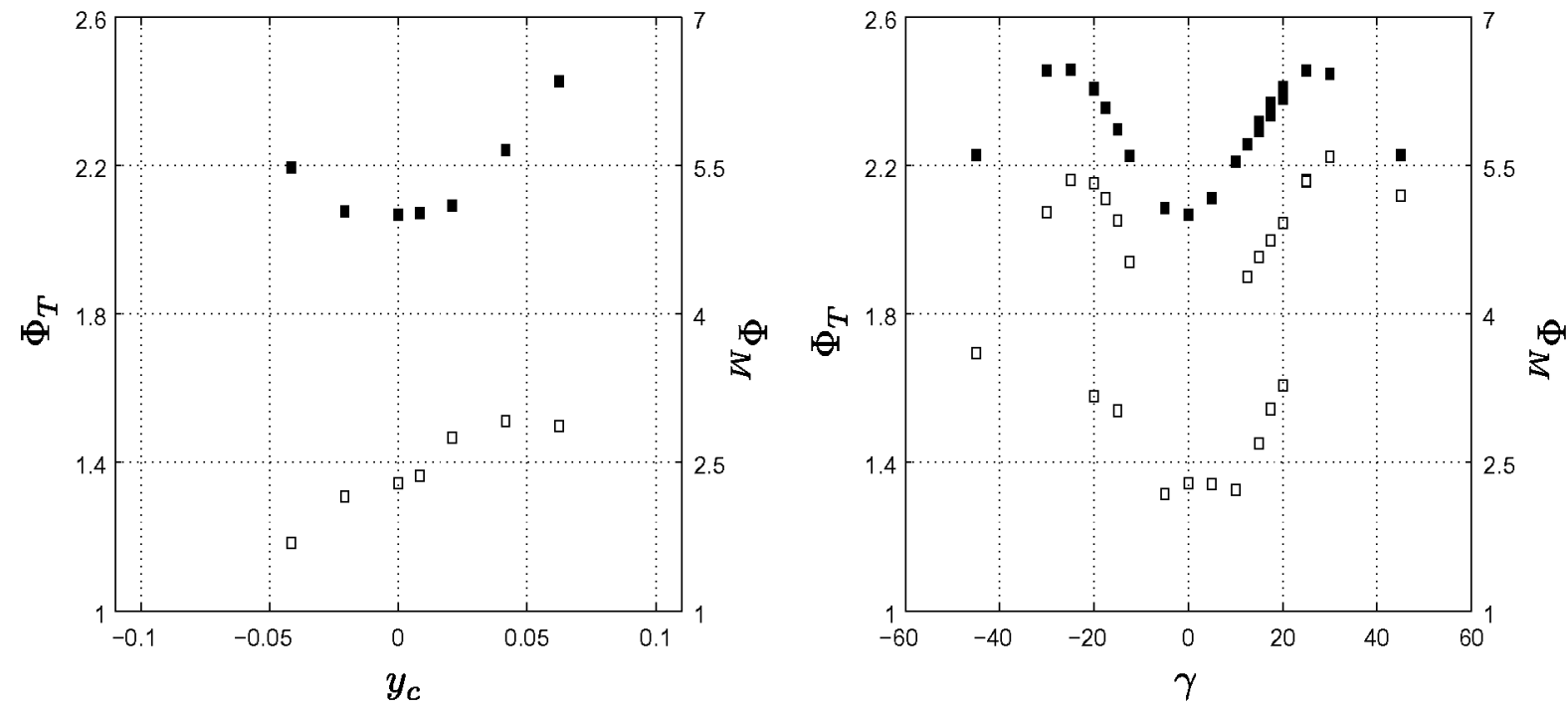

Fig. 9. As in Fig. 7, for $\alpha=1 / 2, \beta=1 / 2$, and $\operatorname{Re}=600$.
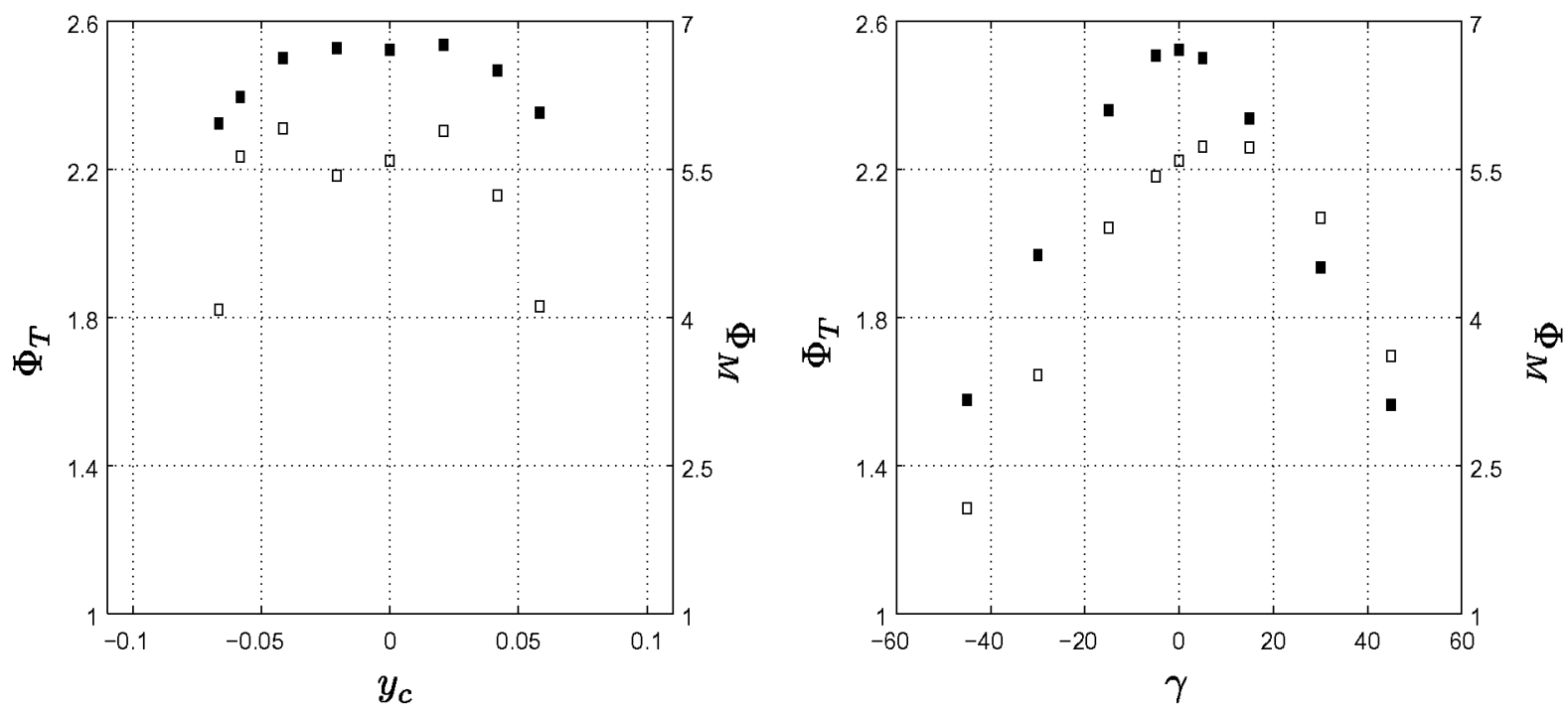

Fig. 10. As in Fig. 7, for $\alpha=1 / 10, \beta=1 / 2$, and $\operatorname{Re}=600$.

tices to the lower wall is offset by a decrease in the strength of the vortices themselves. This is appreciated in the instantaneous vorticity contours Fig. 11, where the upper plot, corresponding to the higher position of the obstacle $\left(y_{c}=1 / 16\right)$ is already an steady one. Both positive and negative obstacle rotation at $\beta=1 / 3$ (Figs. 7 and 8 , right) show a slight improvement in thermal efficiency at $\alpha=1 / 2$, but this deteriorates at $\alpha=1 / 10$.

- The case $\beta=1 / 2$ (Figs. 9 and 10 ) behaves similarly (but with clearer trends) under vertical displacement, but response to rotation is more interesting:

- At $\alpha=1 / 2$ (Fig. 9, right) response to rotation is qualitatively symmetric around $\gamma=0$ (which is due to an approximate symmetry of the whole spatio-temporal pattern, which results from the symmetry $\gamma \rightarrow-\gamma, y \rightarrow 1-y$ of the isothermal fluid dynamical problem, only broken quantitatively by temperature dependence of viscosity) and shows three distinct branches that are now considered for $\gamma>0$. In a first lower branch, which is stable only for $\gamma<10^{\circ}$, thermal efficiency slightly degrades. In an intermediate branch, for $10^{\circ}<\gamma<20^{\circ}$, thermal efficiency clearly improves, while in the upper branch, for $\gamma>12^{\circ}$, a further improvement first occurs, for $\gamma<30^{\circ}$, followed by an opposite trend for larger $\gamma$. Still, the intermediate and upper branches show hysteresis and, what is more important, a jump in thermal efficiency between both branches that does not involve any jump in the mechanical penalty. The reason for thermal efficiency improvement in the intermediate and upper branches is clearly appreciated in the vorticity and temperature contours in Figs. 12 and 13, where it is seen that vortices are stronger, horizontally shorter, and more coherent (which means that the number of vortices and the horizontal damping length are both larger) in the upper branch, which in turn leads to a larger number of stronger thermal plumes.

- At $\alpha=1 / 10$ (Fig. 10 , right) thermal efficiency peaks at $\gamma=12^{\circ}$ (giving $\Phi_{T}=2.2$, approximately the same as its maximum counterpart at $\alpha=1 / 2$ ) and is qualitatively symmetric around this value, suggesting that only the upper branch of the case $\alpha=1 / 2$ survives in this case. 




Fig. 11. Instantaneous (absolute value of) vorticity contours at $\operatorname{Re}=600, \alpha=1 / 2, \beta=1 / 2$, and four positions of obstacle, as indicated.

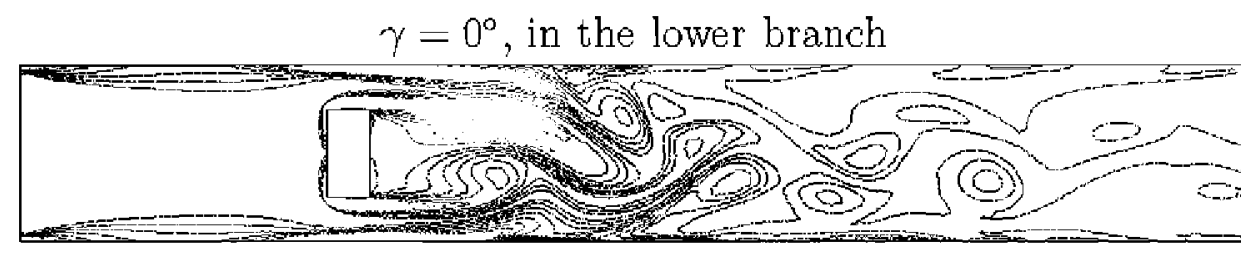

$\gamma=15^{\circ}$, in the intermediate branch
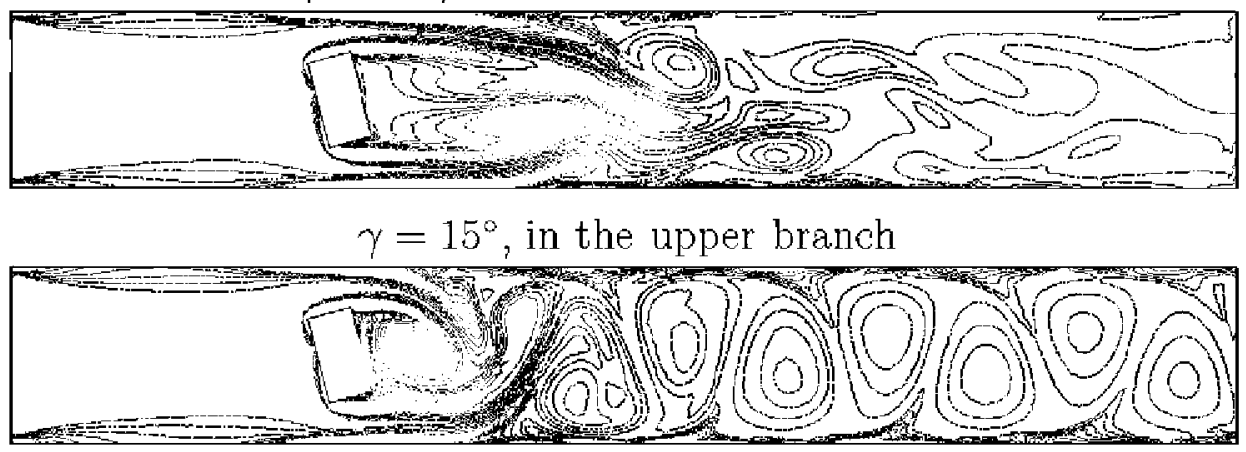

Fig. 12. Instantaneous (absolute value of) vorticity contours at $\operatorname{Re}=600, \alpha=1 / 2, \beta=1 / 2$, and three positive rotations, as indicated.

The results above suggest the following conclusions, which lead obvious qualitative design guidelines:

- Rotation of the obstacle has a dramatic effect in thermal efficiency, which for rectangular obstacles can yield improvements of $120 \%$, both at moderate and small aspect ratios. Comparison of the cases of small and moderate aspect ratios shows that the beneficial effect of the latter, which are in principle not advisable for manufacturing reasons, can also be obtained at moderate aspect ratio rotating the obstacle. Such cooling improvement at moderate aspect ratio results from various flow instabilities, which could be expected in this highly nonlinear problem and have only been outlined above. The main consequence is that such high thermal improvement occurs only in a limited range of rotation angles, meaning that some care must be taken when using this overall result. Complete understanding of the associated thermo-mechanical transitions is well beyond the scope of this paper.

- Vertical displacement instead only yields a weak cooling enhancement, again limited to a short range of vertical displacement. The question is, of course, whether vertical displacement enhances or masks the beneficial effect of rotation, a question 


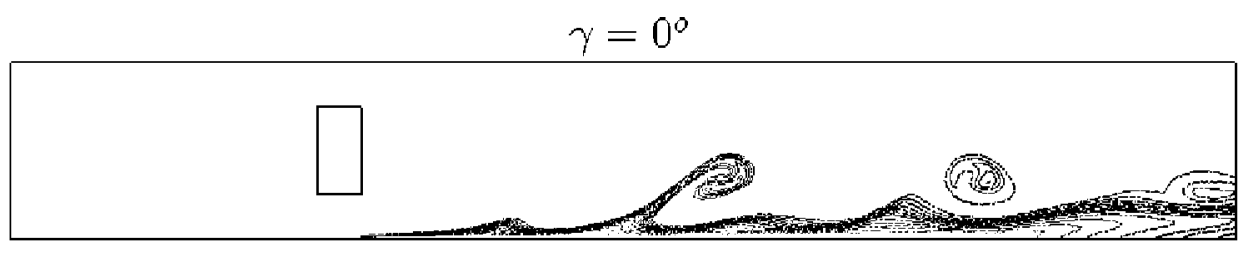

$\gamma=15^{\circ}$ solution on the lower branch

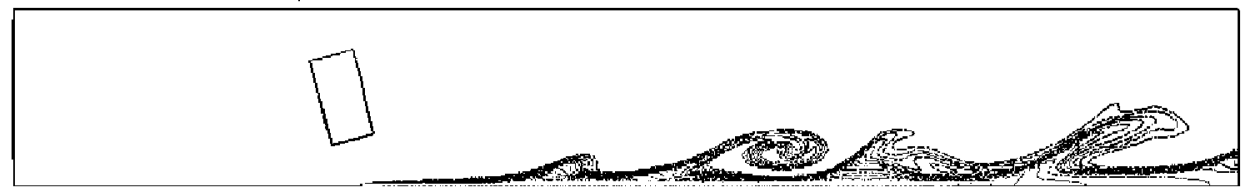

$\gamma=15^{\circ}$ solution on the upper branch

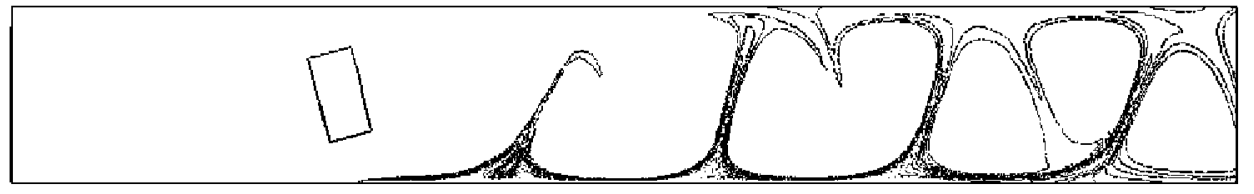

Fig. 13. Instantaneous temperature contours at $\operatorname{Re}=600, \alpha=1 / 2, \beta=1 / 2$, and three positive rotations, as indicated.

that could be also made in connection with the remaining parameters dealing with shape and blockage ratio. We do expect that these affect thermal properties, which depend on the local topology of the flow near the lower part of the obstacle, which in turn is sensible to both local orientation and distance to the lower wall. But again, varying all parameters simultaneously is well beyond performances of standard numerical tools, like the one used in this paper.

\section{Results at higher Reynolds number}

Let us now outline the dependence of the results above as the Reynolds number is increased, in the range $600 \leqslant R e \leqslant 1200$. The upper part of this interval must be looked with care because strong
$3 \mathrm{D}$ effects must be expected well below the upper bound. Thus, the object of this section is just to elucidate the dependence on the Reynolds number of the results above. To this end, thermal efficiency and mechanical penalty are plotted in Figs. 14-16 vs. the Reynolds number for the three configurations considered at low Re, namely circular and $1 / 2$ and $1 / 10$ aspect ratio, rectangular obstacles, which are neither vertically displaced, nor rotated from its symmetric position, at two values of the blockage ratio, $\beta=1 / 3$ and $\beta=1 / 2$. As can be seen in these plots, thermal efficiency either remains constant or slightly increases as the Reynolds number increase, while mechanical penalty always increases. When considering both, thermal and mechanical performances, the circular obstacle at $\beta=1 / 3$ is the best configuration. In any event, increasing the Reynolds number does not seem to be a good
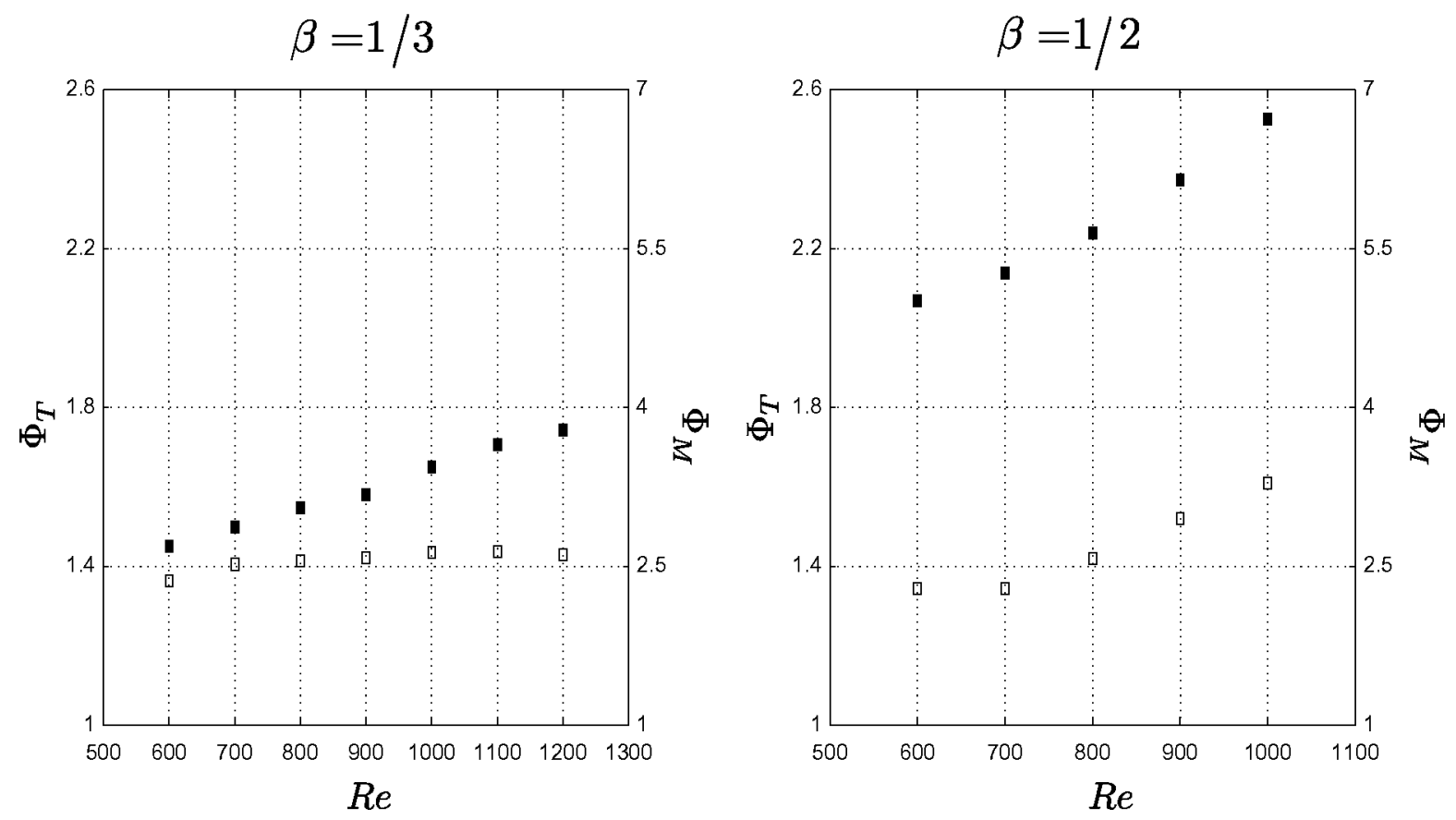

Fig. 14. Thermal efficiency (plain squares) and mechanical penalty (shaded squares) in terms of the Reynolds number for a $1 / 2$ aspect ratio rectangle and the indicated blockage ratio. 

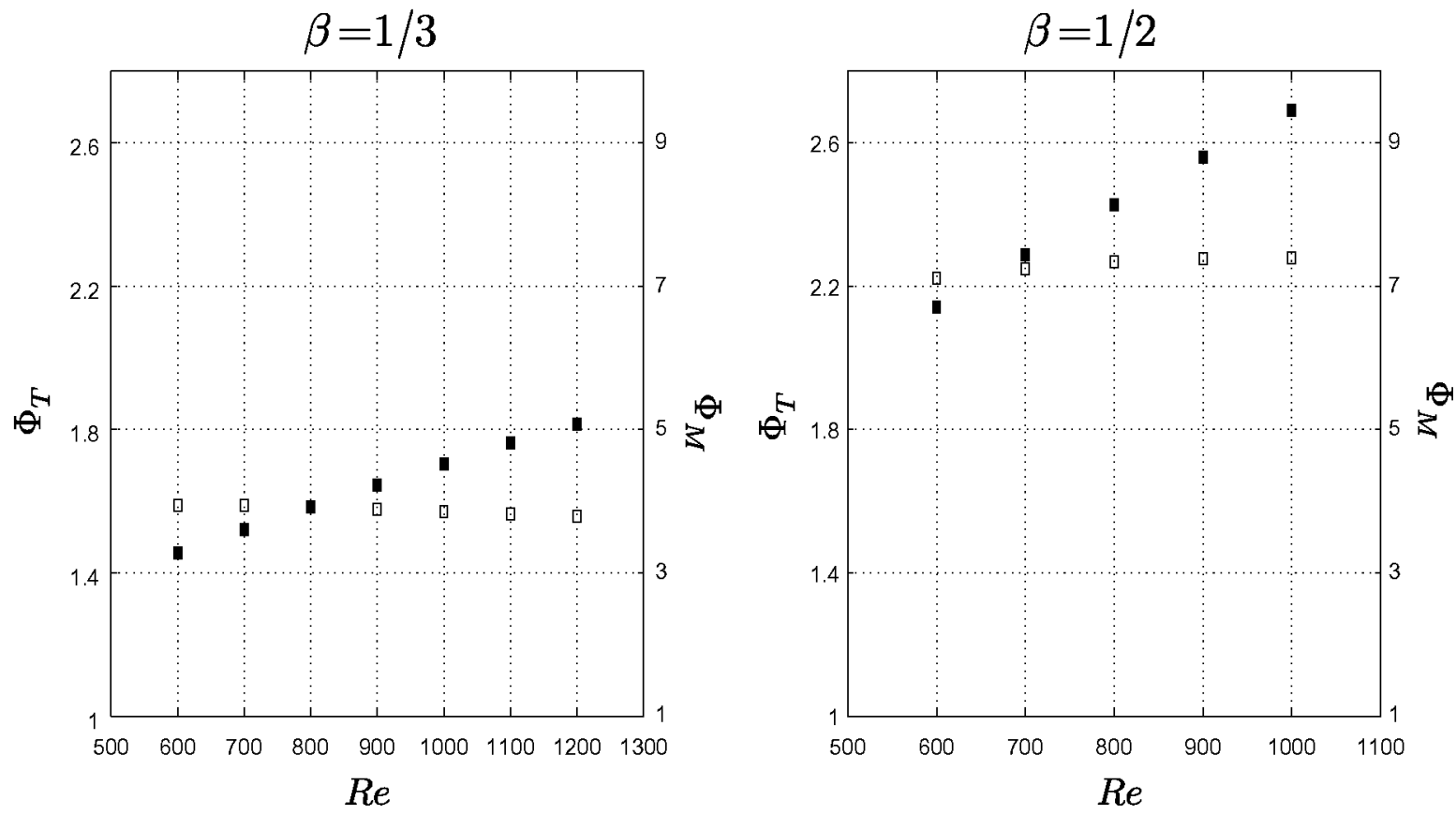

Fig. 15. As in Fig. 14, for the $1 / 10$ aspect ratio rectangular obstacle.
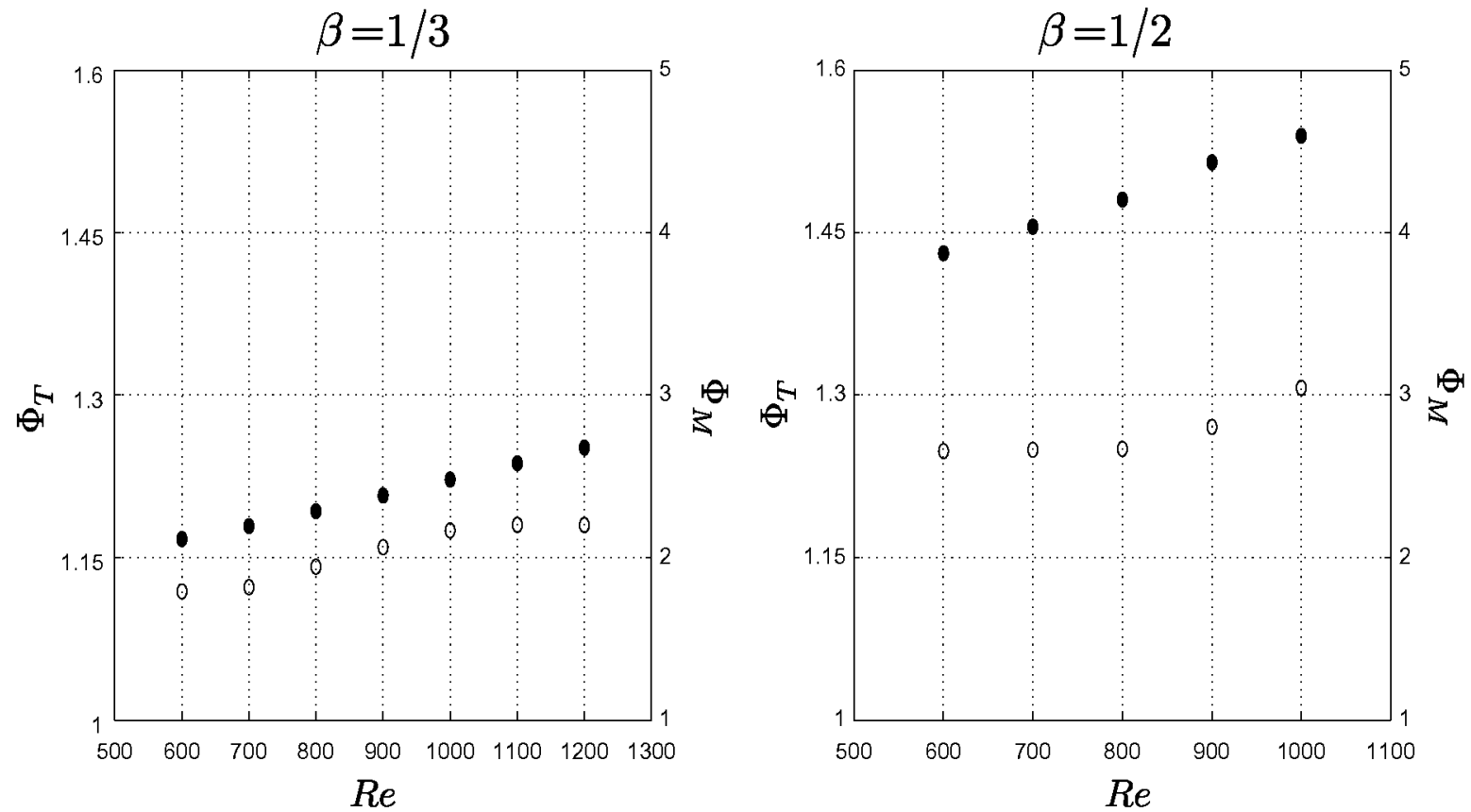

Fig. 16. As in Fig. 14 for a circular obstacle.

way to improve performance, at least if no new ingredients (such as varying the various parameters considered in this paper) are added.

\section{Concluding remarks}

We have analyzed the effect of a vortex promoter in a liquid flow in a channel that is intended to cool a piece of the lower wall. The latter is maintained at a fixed temperature, higher than that of the incoming liquid, the remaining part of the lower wall and the upper wall being thermally insulated (Fig. 1). As a basic configuration, the obstacle is placed at the center of the channel, just above the beginning of nonadiabatic portion of the lower wall. Broadly speaking, it could be said that the use of vortex promoters tends to enhance heat transfer inside micro-channels. However, this enhancement causes a pressure drop and, accordingly, an increase in the needed pump power. Therefore, we have considered both heat transfer from the lower wall, measured by an appropriately defined Nusselt number, and the total pressure drop in the channel, from entrance to exit. Looking for specific, practical guidelines to improve the design of cooling devices, we have varied various parameters, namely the obstacle shape, the blockage ratio, and the obstacle position and orientation, and have selected in most part of the paper a realistic value of the Reynolds number in such 
devices, namely $\operatorname{Re}=600$, although dependence of the Reynolds number up to $\operatorname{Re}=1200$ has been also briefly considered. These are too many parameters to be considered simultaneously using numerical simulations. But preliminary design guidelines can be obtained varying just one parameter at a time, as we have done above. The main conclusions are:

- Regardless of its specific shape, heat transfer increases as the channel blockage ratio increases up to $\beta=1 / 2$ (Fig. 4). The reason for that is the expected one, namely as $\beta$ is increased the initially steady configuration destabilizes and produces a von Karman vortex street that facilitates vertical heat convection from the lower wall, as illustrated in Figs. 2 and 3. Larger blockage ratios increase the pressure drop significantly (Fig. 4); in fact, as it happens in isothermal conditions [18], blockage ratios close to one (not shown in the figures) show a steady configuration that produces a large pressure drop and a low heat transfer benefit. With a centered circular obstacle, heat transfer can be increased by a factor of 1.2 and 1.4 at $\beta=1 / 3$ and $1 / 2$, respectively, the associated mechanical penalty being 2 and 4 , respectively. In fact, in all cases considered above, the relative penalty in pumping power is larger than the heat transfer benefit.

- Better results are obtained varying the obstacle shape. Considering elliptical, rectangular, and triangular obstacles at varying aspect ratios shows that the latter provides best results, then the rectangles, then the ellipses, and that the performance is improved as the aspect ratio is decreased (Figs. 5 and 6). In fact these plots show that, at quite low aspect ratios, the three shapes behave almost identically, which could be expected because geometrical details disappear in this limit, in which they appear to the flow as a slender flat plate. Once these configurations have been considered for reference, practical micromanufacturing reasons advice to disregard both triangles, ellipses and too slender rectangles. $1 / 2$ and $1 / 3$ aspect ratio rectangles seem to be a good compromise, although the $1 / 10$ aspect ratio is also considered for reference.

- Besides the obvious selection of vortex promoter shape and size (blockage ratio), the designer also has the freedom to select its position and orientation. Roughly speaking, we have found out (Figs. 7-10) that thermal efficiency and mechanical penalty depend far more strongly on rotation angle than on position relative to the channel centerline. A new feature is now present, namely that the behavior is no longer monotonic as these two parameters are varied; in fact, some transitions due to flow instabilities between different regimes are now present that yield multiplicity of stable flow configurations in some cases, which is not surprising in this highly nonlinear problem. An example is shown in Fig. 10 (right), which exhibits two transitions that yield three flow regimes. Transition between the intermediate and upper branches in this figure involves a significant jump in thermal effectiveness that, fortunately enough, is not accompanied by any jump in the pressure drop. The difference between the three regimes is appreciated in the vorticity and temperature contours in Figs. 12 and 13. Now, it is precisely looking for this type of behavior that motivated this work at the very beginning, from our believing that a good way to get optimal design solutions of these devices is to seek geometric configurations that are as simple as possible, but promote nonlinear flow instabilities that enhance vertical convection.

- The brief analysis of variation of thermal and mechanical efficiencies with the Reynolds number for the most basic configurations (Figs. 14-16) shows that increasing Re has only a slight benefit if any.

- When looking at the implementation of vortex promoters on practical engineering designs, micro-manufacturing aspects should be accounted for. In this regard, a simple and safe guideline is that the $1 / 2$ aspect ratio rectangle rotated $30^{\circ}$ clockwise represents a good compromise, as explained above.

- An obvious question arises, as already mentioned at the end of Section 3, namely how do the results above improve or deteriorate as all parameters are varied simultaneously. Answering this is well beyond the scope of this paper, and requires some more sophisticated mathematical tools than the one used in this paper, namely numerical simulations on the time dependent problem, eliminated transient behaviors, which both are robust and ensures (nonlinear) stability of the final (time dependent in general) state. Both numerical continuation methods (which are nontrivial when applied to time dependent states in a many degrees of freedom system) and reduced model equations based on proper orthogonal decomposition, Galerkin projection can be used to give a reasonable answer to this question,which is currently under research.

\section{Acknowledgements}

This research was partially supported by the Spanish Ministry of Education and Science, under Grants MTM2007-67596-C02-02 and CSD2006-00032 (M.M. and F.V.), DPI2005-05572 (A.V.), and TRA2007-65699 (J.M.V.).

\section{Appendix A}

Here, we address the COMSOL Multiphysics solver, testing a series of configurations that are relevant to the problem being considered. Also, a mesh sensitivity analysis is performed.

In order to validate the Taylor-Hood $P_{2}-P_{1}$ Navier-Stokes solver, we consider an open $2 \mathrm{D}$ flow around a circular obstacle, at a Reynolds number (based on the diameter) of 150, using a discretization of 100,000 degrees of freedom in a computational domain of $30 \times 20$ diameters. The computed Strouhal number, 0.186 , is very close to available experimental correlations (a measured Strouhal number of 0.1834 is reported in [21]).

Coupling of the Navier-Stokes solver with a $P_{2}$ discretization of the energy equation (with $P_{1}$ Lagrange multipliers to compute of the Nusselt number) is checked considering heat transfer from a hot circular obstacle in an open $2 \mathrm{D}$ flow, with a variable Prandtl number (corresponding to air) and the same Reynolds number as above. Now, a discretization with 125,000 degrees of freedom is used. A two terms, least square approximation of $\mu(T)$ and $k(T)$ (computed from data in [22]) is used. Inlet and obstacle temperatures are 297 and $347 \mathrm{~K}$, respectively. The computed Nusselt number, 6.01, agrees reasonably well with experimental correlations reported in [23], namely 6.27 and 6.30 , with a reported accuracy of $\pm 4 \%$.

Once COMSOL Multiphysics solvers have been tested, we check that results are mesh independent. This is made testing our implemented meshing guidelines at a large value of the Reynolds number (in fact, larger than any case considered in this paper). Unstructured meshes are generated (i) specifying mesh sizes on every boundary (typically, element lengths below $1 / 20$ th of obstacle diameters are taken near the obstacle boundary, while sizes of $1 / 200$ th of the hydraulic diameter are considered near the nonisothermal channel wall) and (ii) performing a latter refinement on a region near the boundary layer attached to the nonisothermal wall (to ensure not only that this region is well resolved but also that no thermal artificial diffusion is needed there). According to all these, meshes made up of about 100,000 degrees of freedom are generated (only a $10 \%$ corresponding to local refinement). A generic view and two close-up views (near obstacle and wall regions) of the mesh are presented in Fig. 17. 

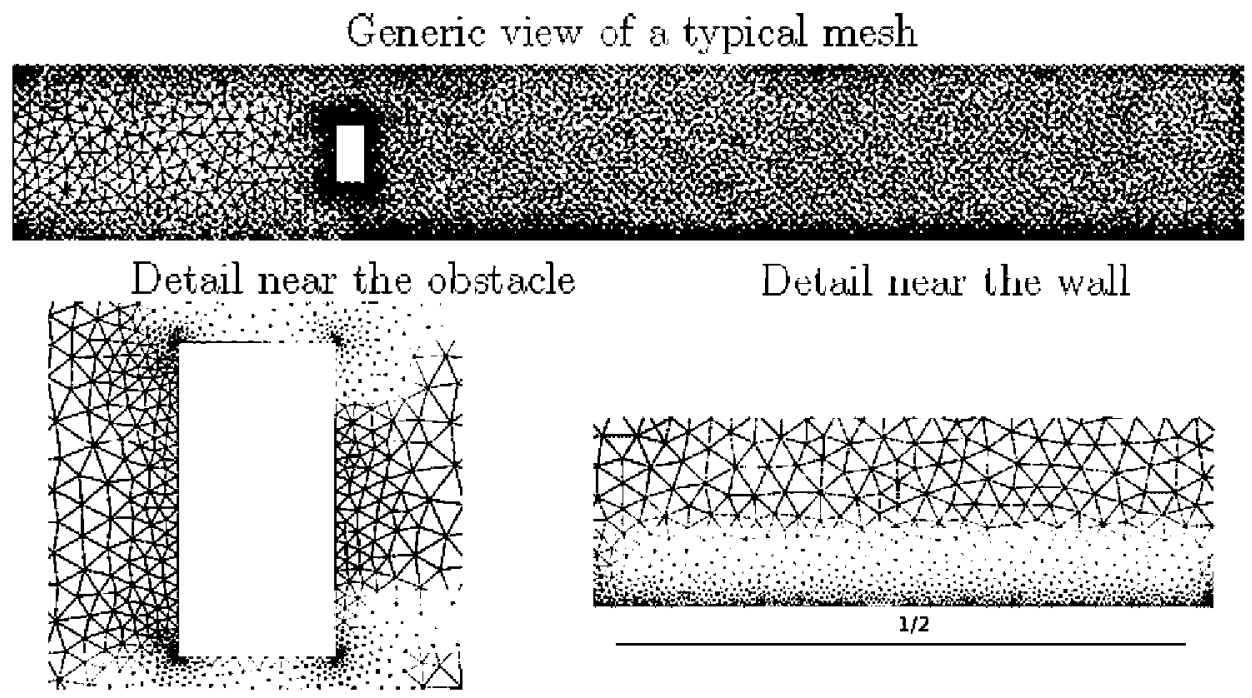

Detail near the wall

Fig. 17. Overview and some details of a typical computational domain.

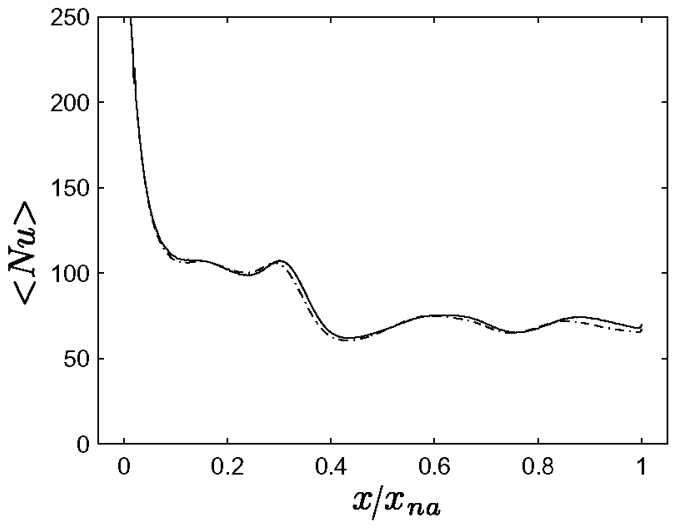

Fig. 18. Time averaged, local Nusselt number $\langle\mathrm{Nu}\rangle$ vs. horizontal position for (i) the mesh generated with the indicated meshing parameters (-) and (ii) a globally refined mesh (-.-).

Then, we solve the problem considered in the paper, with a $1 / 2$ aspect ratio rectangular obstacle, at Reynolds 3000 and a $1 / 3$ blockage ratio, using both the mesh generated with the above mentioned guidelines and a second mesh, obtained with a global refinement that doubles the number of degrees of freedom up to 220,000. Results are presented in Fig. 18, where the time averaged, local Nusselt number on the nonadiabatic part of the lower wall is plotted vs. horizontal position, as obtained with the two mentioned meshes. As can be seen, no significant improvement results from global refinement.

\section{References}

[1] M. Rahnama, H.H. Moghaddam, Numerical investigation of convective heat transfer in unsteady laminar flow over a square cylinder in a channel, Heat Transfer Eng. 26 (10) (2005) 21-29.

[2] S. Turki, H. Abassi, S.B. Nasrallah, Two-dimensional laminar fluid flow and hea transfer in a channel with a built-in heated square cylinder, Int. J. Thermal Sci. 42 (2003) 1105-1113.

[3] T. Icoz, Y. Jaluria, Design optimisation of size and geometry of vortex promoter in a two-dimensional channel, J. Heat Transfer 128 (2006) 1081-1092.
[4] H. Abbasi, S. Turki, S.B. Nasrallah, Numerical investigation of forced convection in a lane channel with a built-in triangular prism, Int. J. Thermal Sci. 40 (2001) 649-658.

[5] H. Abbasi, S. Turki, S.B. Nasrallah, Mixed convection in a plane channel with a built-in triangular prism, Numer. Heat Transfer A 39 (2001) 307-320.

[6] S. Nitin, R.P. Chhabra, Non-isothermal flow of a power law fluid past a rectangular obstacle (of aspect ratio $1 \times 2$ ) in a channel: drag and heat transfer, Int. J. Eng. Sci. 43 (2005) 707-720.

[7] A. Valencia, Turbulent flow and heat transfer in a channel with a square bar detached flow the wall, Numer. Heat Transfer A 37 (2000) 289-306.

[8] G. Papaioannou, D.K.P. Yue, M.S. Triantafyllou, G.E. Katniadakis, Threedimensionality effects in flow around two tandem cylinders, J. Fluid Mech. 558 (2006) 387-413.

[9] S. Kang, Characteristics of flow over two circular cylinders in a side-by-side arrangement at low Reynolds numbers, Phys. Fluids 5 (9) (2003) 2486-2498.

[10] Y. Tasaka, S. Kon, L. Schouvelier, P. Le Gal, Hysteretic mode exchange in the wake of two circular cylinders in tandem, Phys. Fluids 18 (2006). 084104-1-6.

[11] J. Niu, Z. Zhu, S. Huang, Numerical study of convective heat transfer from two identical square cylinders submerged in a uniform cross flow, Numer. Heat Transfer A 50 (2006) 21-44.

[12] A. Valencia, R. Paredes, Laminar flow and heat transfer in confined channe] flow past square bars arranged side by side, Heat Mass Transfer 39 (2003) 721-728.

[13] A. Valencia, M. Sen, Unsteady flow and heat transfer in plane channels with spatially periodic vortex generators, Int. J. Heat Mass Transfer 46 (2003) 3189 3199 .

[14] A. Valencia, W. Calderón, Convective heat transfer in plane channels with inline mounted rectangular bars, Int. J. Numer. Methods Heat Fluid Flow 14 (7) (2004) 866-878

[15] G.M. Laskowski, S.P. Kearney, G. Evans, R. Greif, Mixed convection heat transfer to and from a horizontal cylinder in cross-flow with heating from below, Int. J. Heat Fluid Flow 28 (3) (2007) 454-468.

[16] F.P. Incropera, D.P. DeWitt, Introduction to Heat Transfer, John Wiley \& Sons, New York, 1996.

[17] COMSOL Multiphysics 3.3, COMSOL AB, 2007. Available from: <http:/ www.comsol.com>.

[18] M. Sahin, R.G. Owens, A numerical investigation of wall effects up to high blockage ratios on two-dimensional flow past a confined circular cylinder, Phys. Fluids 16 (5) (2004) 1305-1320.

[19] C.H.K. Williamson, Vortex dynamics in the cylinder wake, Annu. Rev. Fluid Mech. 28 (1996) 477-539.

[20] S. Mittal, S. Singh, Vortex-induced vibration at subcritical Re, J. Fluid Mech. 534 (2005) 185-194

[21] H. Persillon, M. Braza, Physical analysis of the transition to turbulence in the wake of a circular cylinder by three-dimensional Navier-Stokes simulation, J. Fluid Mech. 365 (1998) 23-88.

[22] R.W. Johnson (Ed.), The Handbook of Fluid Dynamics, CRC Press, Boca Raton, 1998 .

[23] A. Sharma, E. Soehngen, Heat and fluid flow across a square cylinder in the two-dimensional laminar flow regime, Numer. Heat Transfer A 45 (2004) 247 269. 